\title{
Superconducting Magnets for the LHC
}

\author{
R. Perin describes CERN's collaboration with industry for the development \\ of high-field superconducting magnets for the LHC
}

\begin{abstract}
Building an accelerator ring $27 \mathrm{~km}$ in circumference occupied by $24 \mathrm{~km}$ of superconducting magnets, refrigerated largely with superfluid helium, weighing altogether 36000 tonnes, and producing a magnetic field that is at least $50 \%$ higher than the field for any likely predecessor, and all this within the confines of an existing tunnel, is a daunting, but exhilarating, task. CERN has taken up the challenge in proposing the Large Hadron Collider (LHC) that would exploit the existing LEP tunnel (Fig. 1) and facilities in pushing research in high energy physics to the limit.
\end{abstract}

Synchrotron accelerator rings remain the most efficient machines for accelerating and colliding high energy, high intensity beams of protons. Progress requires ever increasing beam energies and since the attainable energy is directly proportional to the bending magnetic field, CERN envi- sages raising the nominal operating field to $8-10 \mathrm{~T}$, corresponding to $6.4-8.0 \mathrm{TeV}$ per beam. The HERA ring now under construction at DESY near Hamburg is aiming for 4.7 $T$ with perhaps an eventual increase to 5 or 5.5 T, while the SSC ring, for which detailed design work and site preparation started last year in Texas, is based on using 6.6 T and a completely new facility.

\section{Magnet layout}

The LHC design envisages two counterrotating proton beams circulating in separate vacuum tubes made up of eight, $2.8 \mathrm{~km}$ long, circular arcs linked by $0.5 \mathrm{~km}$ long, roughly straight sections. One half of each $50 \mathrm{~m}$ standard cell that would be repeated along each arc consists of four superconducting dipole magnets ( $9.5 \mathrm{~m}$ long) and a short straight section containing a main quadrupole magnet for focussing each

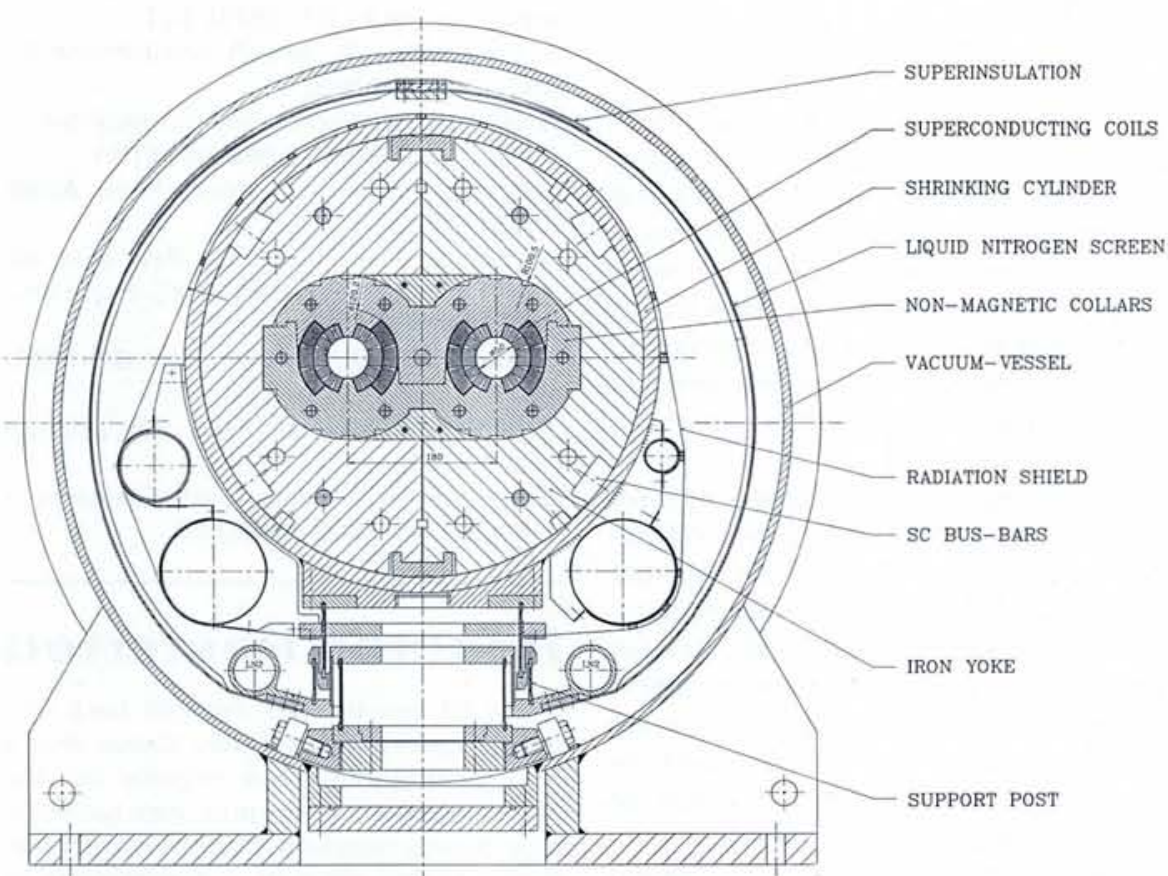

Fig. 2 - Cross-section illustrating the "hybrid mechanical" design of a superfluid helium cooled, $10 \mathrm{~T}$, twin aperture dipole magnet for the LHC $11.35 \mathrm{~m}$ long models of which are now being fabricated by industry). The four sets of superconductor coils are clamped in place at room temperature with prestressed collars restained using specially designed pins. The outer shrinking cylinder - which also acts as the external wall of the pressurised container for the superfluid helium cryogen which permeates the coils - contracts during cool down, pulling the two halves of the yoke together. At the operating temperature, compressive forces in the yoke counteract radial electromagnetic forces in the coils. The two-layer coils are wound with close to a Roman arch construction using mechanically stable, high aspect ratio superconducting cables based on NbTi alloys. The cross-section also shows various channels for cryogens and power cables, as well as shields and the $0.92 \mathrm{~m}$ diameter outer vacuum vessel.

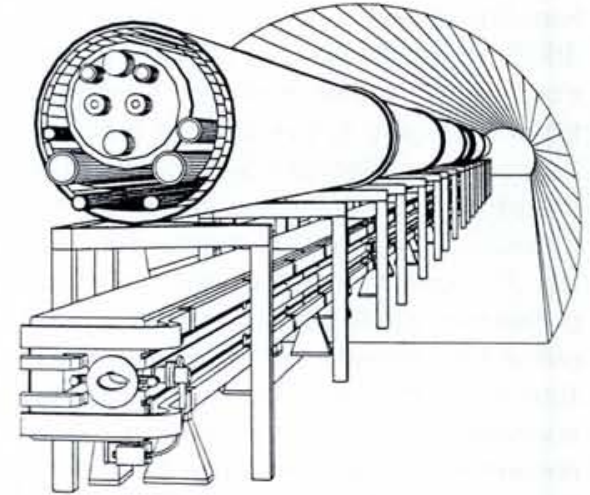

Fig. 1 - Schematic view of a section through the LEP tunnel at CERN, Geneva illustrating the proposed LHC accelerator, comprising primarily dipole superconducting magnets together with some superconducting quadrupoles and multipole corrector magnets, mounted above the existing LEP machine. The section shows the twin apertures of a dipole magnet together with the outer wall of the cryostat and ducts for cryogens.

beam, a second quadrupole for fine tuning, and a combined sextupole/dipole magnet. Owing to the limitation of space, the dipoles and focussing quadrupoles would have separate apertures and superconducting field coils for the beam lines, but the iron yoke and the cryogenic vacuum vessel would be shared, along with cryogen channels and power circuits (Fig. 2). The remaining magnets would have the conventional single-aperture design.

The apertures are $50 \mathrm{~mm}$ in diameter and their separation $(180 \mathrm{~mm})$ in the twin aperture dipoles and quadrupoles is determined by the need to have magnetically uncoupled corrector magnets.

\section{Superconductors}

The bulk of the magnet coils would be wound from superconducting cables based on filaments of a niobium-titanium alloy in a copper matrix. These cables are now commercially available in large quantities. However, the operating temperature would be reduced from the conventional $4.2 \mathrm{~K}$ to below $2 \mathrm{~K}$ by using superfluid helium instead of the normal liquid helium as the cryogen. This permits the current density in the conductor to be increased by a factor of about three to achieve a compact, high field, design. 


\section{Persistent currents}

Calculations have shown that distortions introduced by persistent circulating currents generated when the coil excitation is changed are roughly proportional to the filament diameter. These distortions have proved to be one of the most important factors limiting the performance of colliders built using superconducting magnets. CERN, following a cost-benefit analysis, has selected a diameter of $\leq 6$ microns.

Compact coils designs, vital for lowering costs and allowing for space limitations, will also greatly reduce field distortions by minimizing the amount of conductor in the coil windings.

\section{Superfluid helium cryogenics}

The use of a superfluid helium cryogenics on such a large scale would probably represent the first of its kind. Technical complications seem surmountable in view of experience gained with operating the superfluid cooled Tore Supra tokamak reactor in France that was designed to explore fusion under long pulse conditions. The additional capital cost of the cryo-magnet system is estimated to be only $8-10 \%$ relative to the conventional liquid helium approach, and the additional power consumed in maintaining $2 \mathrm{~K}$ instead of $4.2 \mathrm{~K}$ (refrigeration costs being the most important running cost) should be about $20 \%$.

Impregnating the specially designed porous structure of the magnet coils with superfluid helium having a low viscosity, a high thermal conductivity and a high heat capacity should yield excellent stability of magnet operation in the presence of the extremely intense beams circulating in the LHC.

\section{Industrial Collaboration Gets Underway}

CERN realised right from the start that it would be fully occupied commissioning the LEP machine so it would have to mobilise all available external resources to carry out essential development work for the LHC. In the case of the high-field beam magnets, collaboration with industry began in 1985 with a meeting of representatives from the superconductor and electrical engineering industries. The requirements were described and by 1986 , Ansaldo had accepted a proposal to build two $8 \mathrm{~T}$ model dipoles of $1.35 \mathrm{~m}$ length with a single aperture, but using superfluid helium as the cryogen. The first magnet, delivered in 1988 for testing at CERN and later at CEN, Saclay, attained a maximum central field of $9.3 \mathrm{~T}$; the second, using stronger insulation, reached $9.45 \mathrm{~T}$ in July 1989.

CERN has had considerable experience with superconducting magnets and was the first to use them in an accelerator when it designed and built a high gradient, large aperture prototype quadrupole in 1976 for high luminosity insertion in the ISR ring. Eight of the nine magnets subsequently ordered from industry operated successfully until the ring was shut down. There followed in 1984 a $6 \mathrm{~m}$ long, $4.5 \mathrm{~T}$, NbTi

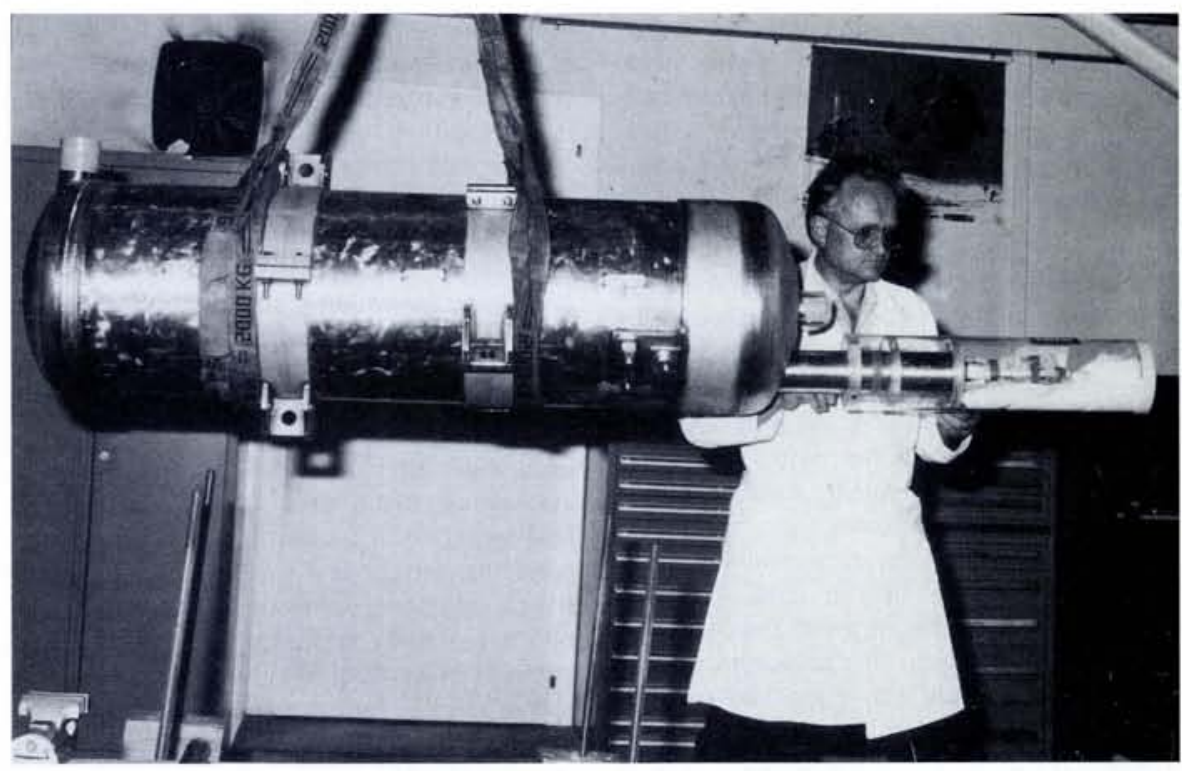

Fig. 3 - The first $1.35 \mathrm{~m}$ long, superfluid helium cooled $8 \mathrm{~T}$ model dipole for the LHC manufactured by Ansaldo for CERN in 1988. The magnet attained 9.3 T in tests. Visible is the stainless steel shrinking cylinder that formed the main part of the helium cryostat.

prototype dipole that resembled the dipoles now being installed at HERA. Several small magnets for transferring beams have also been built and a series of insertion quadrupoles for LEP designed in 1984 were manufactured industrially.

\section{Split yokes}

The success of the Ansaldo $8 \mathrm{~T}$ models and CERN's ability to continue directing magnet development stems from its pioneering of an elegant solution to a very serious problem. Superconducting coils for magnets must be kept in place against tremendous electromagnetic forces by a retaining solid structure when the coils are energised. Any distortion or movement affects the field quality and may even induce a premature "quench" or transition when the coil stops superconducting and reverts to the normal state. Conventional designs, involving only prestressed collars placed around the coils, are not viable at the high field strengths required for the LHC since the appropriate prestresses exceed the creep strengths of parts of the structure during unavoidable storage of uncooled magnets at room temperature.

It had been appreciated for sometime that an efficient mechanical design would require some contribution towards support by components other than the collar. In 1985, R. Perin, head of superconductor magnet development at CERN, proposed a "hybrid mechanical" design where the iron yoke is split vertically (Fig. 2). The structure contracts during cool down and at some predetermined temperature the gap in the yoke closes, whereupon compressive stresses in the yoke start to build up. At the operating temperature, these stresses balance outward electromagnetic forces in the energised coils.

Recent detailed calculations show that the approach is viable up to fields of about $11 \mathrm{~T}$. Measurements of the Ansaldo ma- gnets have confirmed the value of the new design at $9 \mathrm{~T}$ : compressive stresses in the coil are roughly $50 \%$ of those that would be expected for the conventional approach. Its main disadvantage is the need for more precise manufacturing and assembly of the structural components.

\section{Latest Developments}

The cooperation with Ansaldo heralded a sharp increase in CERN's collaboration with industry. Arrangements have remained similar in each case: CERN provides preliminary design data, superconductor cable, technical assistance, some facilities and final testing. The industrial partner generally contributes design staff who work at CERN, tooling, materials other than superconductor, magnet fabrication, and assistance during final testing.

\section{$\mathrm{Nb}_{3} \mathrm{Sn}$ conductors}

Conductors based on a niobium-tin compound offer current densities at $4.2 \mathrm{~K}$ similar to those for NbTi based conductors operating at $2 \mathrm{~K}$ as well as a higher field capability. However, they are much more difficult to fabricate and their commercial availability is extremely limited. Nevertheless, CERN realised that certain parts of the LHC ring, where heat dissipation from the beam would be unusually high (e.g. crossing points), need high-field NbSn magnets operating at $4.2 \mathrm{~K}$. They would be capable of supporting sudden heat loads and higher fields in the windings with a large safety margin. A collaboration with Elin-Union was therefore launched in 1986 to design and build a $1 \mathrm{~m}$ long, 9-10 T, single aperture model dipole using $\mathrm{NbSn}$ conductor. A halfbore coil reached $10.2 \mathrm{~T}$ at $4.3 \mathrm{~K}$ in a magnetic mirror facility and the full-bore magnet attained $9.5 \mathrm{~T}$ in tests at CERN in 1989.

The development of NbSn dipoles continues with a Dutch collaboration comprising Holec, the University of Twente, FOM 
and NIKHEF that is building a $1 \mathrm{~m}$ long, $10 \mathrm{~T}$, twin-aperture model. Design work started in 1989 and the aim is to exploit the hybrid mechanical design, together with a high-performance conductor developed in the Netherlands by the ECN.

\section{Keystoning and Roman arches}

Conventional superconducting cables for beam line magnets have approximately rectangular cross-sections with high aspect ratios. They are made by wrapping together 20-40 gently spiralling superconductorcopper composite wires with the wellestablished "Rutherford" configuration, named after its developers.

Compact, small aperture coils can be wound with close to the ideal "Roman arch" structure if the edges of the cable are angled or keystoned. This structure, where the insulated cable turns are laid up one upon another in an arc, ensures that radial inward contraction of the yoke and collar is transferred into azimuthal stresses in the coil that counteract any tensile forces that may be generated on energising the coils. Being mechanically weak, the interturn insulation cannot support significant tensile strain without the formation of small cracks which may induce premature quenches or severely impair the magnetic performance.

\section{Aspect ratios}

Increasing the aspect ratio of the cable improves the mechanical stability of the coil. It also reduces manufacturing costs by allowing a two layer design where three layers may be otherwise required. However, it is only recently that cable manufacturing techniques, stimulated by the LHC requirements, have advanced to the point that industry can supply high aspect ratio cable with large keystone angles. The Ansaldo $8 \mathrm{~T}$ models were wound using cable with an aspect ratio of about 8.5. This is being raised by $35 \%$ for the Dutch models, and for the four twin-aperture models discussed below.

\section{Aluminium versus stainless steel}

Preparations for the final stage of the dipole model building programme were launched in 1988 and completed last year by the finalisation of contracts between CERN and four separate companies for the supply, starting in 1990, of four variants of a twin-aperture, $1.35 \mathrm{~m}$ long, $10 \mathrm{~T}$, NbTi model dipole for the LHC (see Fig. 2).

The basic design remains essentially the same as for the single-bore models built by Ansaldo. However, in three cases (AnsaldoABB, Holec and Jeumont-Schneider) the coils of the two apertures are restrained using common collars, while in the fourth (Elin-Union) each aperture has its own separate collar system.

The collars are in all four cases made using a high strength aluminium alloy. Compared to designs based on stainless steel, this offers lower cost, the advantage of being non-magnetic, and a high thermal contraction on cool-down, thus minimizing prestresses at room temperature.

The model made by Ansaldo-ABB will use a stainless steel shrinking cylinder while the remainder will use cylinders made from an aluminium-magnesium alloy having a higher thermal contraction to give improved performance through reduced prestressing.

However, the LHC team plans to use the shrinking cylinder as the outer wall of the helium vessel which must be vacuum tight. Aluminium is notoriously difficult to join and repair by welding so the choice for the cylinder material remains undecided.

\section{Working with superfluid helium}

Delivery is expected shortly of a $10 \mathrm{~m}$ long, twin aperture 7.5 T prototype of the LHC dipole, designed at CERN, from ABB in Manheim, FRG. Since the cost of a prototype beam magnet is mainly determined by the tooling cost, it was decided to save time and expense by winding the coils with the same configuration as the one being used since 1987 for manufacturing HERA's dipoles (most of which have been delivered). The coil aperture is $75 \mathrm{~mm}$ but the rest of the structure corresponds to the LHC design.

The objective is to test the viability of pressurised superfluid helium cryogenics as this has never been done using a horizontal cryostat of the length needed for an LHC dipole magnet. FBM in Italy will mount the dipole in a cryostat and deliver it this spring to a test facility at CEN, Saclay. If all goes well, it is hoped to eventually insert the prototype dipole in a section coupled to CERN's SPS accelerator ring to evaluate thermal stability under simulated beam losses.

\section{... and soon a standard cell?}

Two $10 \mathrm{~m}$ long, $10 \mathrm{~T}$, twin aperture, $\mathrm{NbTi}$ prototype dipoles have been ordered by the INFN in Italy. CERN hopes to order two more fairly soon, followed by a further six next year. Although the design of the LHC dipoles is not yet finalised, the objective is to mount these magnets in a prototype of the $100 \mathrm{~m}$ long standard cell for the LHC in 1992.

\section{Quadrupoles}

Every half-cell will also require a $3 \mathrm{~m}$ long twin-aperture quadrupole for focussing the beams, and a shorter (1 $\mathrm{m}$ long) twin-aperture quadrupole for fine tuning. The main quadrupoles pose a significant technical challenge since not only will they use as yet untried superfluid helium cryogenics, but to ensure an unprecedented level of focussing in the tight confines of the LEP tunnel, they will also each have a gradient of the magnetic field that is about 2.5 times larger than for HERA (250 T/m as opposed to $90 \mathrm{~T} / \mathrm{m}$ ). The novel twin-aperture instead of the usual single aperture configuration will also be adopted. The focussing quadrupoles are being developed in collaboration with CEN, Saclay.

The overall design of the tuning magnet resembles the ISR's made in the 1970's. It will use a similar, though smaller, conductor and a reduced aperture. The performance requirements $(120 \mathrm{~T} / \mathrm{m})$ imply that the new magnet will have a different mechanical structure.

\section{Spain steps forward}

In September 1988, at a CERN accelerator school in Salamanca, Sercobe, representing Spanish companies specialising in building industrial equipment, indicated an interest in collaborating. Five months later, two designers from Spain supported by Sercobe and the CDTI were hard at work at CERN with the LHC magnet development team designing a prototype of the tuning quadrupole. The design was finalised by the end of the year.

An agreement with the so-called ACICA industrial consortium comprising Abengoa, Canzler, Indar, Cenemesa and AME was signed in June 1989 so that magnet construction could start in early 1990. Canzler will meanwhile fabricate a vertical superfluid helium cryostat for testing the prototype. All this activity represents an important "first" for Spanish industry which up to now has never been involved to any significant extent in the construction of superconducting electromagnetic equipment.

\section{Multipole Correctors}

The LHC design calls for combined sextupole/dipole and sextupole/octupole/decapole corrector magnets in each cell of the circular arcs to eliminate beam instabilities and to compensate for field distortions in the main dipoles and quadrupoles. It is planned to use superconducting single aperture versions, one for each beam, and a collaboration with Tesla Engineering and the Rutherford-Appleton Laboratory in the UK was initiated in 1988 for the design and construction of a $1.1 \mathrm{~m}$ long prototype of the sextupole/dipole magnet. The prototype is in the final stage of manufacture and will probably be tested later in the year.

As with the other magnets, the mechanical design of this single bore, correcting multipole magnet is based on the CERN concept of the controlled interplay of prestressing and thermal contraction, so the organization remains once again heavily involved with the transfer of know-how and the provision of the main drawings. In line with other collaborations, Tesla is charged with the rest of the manufacturing steps except for provision of the NbTi superconductor cable which remains one of CERN's responsibilities.

\section{The Future}

CERN has also launched collaborations with industry aimed at various items connected with high-field superconducting magnets for the LHC. These include special high current diodes, improved insulation systems, cryogenic current leads and cryostat design. Coordination of this work is concentrated in a single team headed by $R$. Perin following the recent reorganization of CERN with the successful commissioning of LEP. All will be anxious to see if CERN's two track, twin aperture approach, following both the $\mathrm{NbTi}$ and the $\mathrm{NbSn}$ alternatives for the superconductor can help keep Europe as the leader in high-field accelerator magnet technology and high energy particle physics. 